\title{
Evaluation of a recombinant DNA hepatitis $B$ vaccine in a vaccinated Nigerian population
}

\author{
Moses P. Adoga1', Grace Pennap¹, Becky O. Akande", Jamey P. Mairiga², Simon Pechulano ${ }^{3}$, Simon M. \\ Agwale $^{4}$ \\ ${ }^{1}$ Microbiology Unit, Department of Biological Sciences, Faculty of Natural and Applied Sciences, Nasarawa State \\ University, P. M. B. 1022, Keffi, Nigeria \\ ${ }^{2}$ Department of Biochemistry and Molecular Biology, Faculty of Natural and Applied Sciences, Nasarawa State \\ University, P. M. B. 1022, Keffi, Nigeria \\ ${ }^{3}$ Innovative Biotech Ltd Keffi, Nasarawa State, Nigeria \\ ${ }^{4}$ Innovative Biotech USA Inc, Frederick Innovative Technology Center, Frederick MD 21704, USA
}

\begin{abstract}
Introduction: Recombinant hepatitis B vaccine was introduced in 1986 and has gradually replaced the plasma-derived hepatitis B vaccine. No published data are available on the immunogenicity of hepatitis B vaccines in Nigerians. The current study aimed to evaluate protective seroconversion rates after vaccination with Shanvac-B rDNA hepatitis B vaccine in Nigerian subjects between January and September 2009.

Methodology: After having obtained informed consent and ethical clearance, $2 \mathrm{~mL}$ of blood were aseptically collected from each participant aged $\leq 50$ years, one month after the first, second and third doses of the vaccine. Sera were separated into cryovials and frozen at $-21^{\circ} \mathrm{C}$ until analysed for the detection of the protective antibody titre induction. Protective antibody titre was defined as a titre of $\geq 10 \mathrm{mIU} / \mathrm{mL}$.

Results: Of the 376 participants, $192(51.1 \%)$ were males and 184 (48.9\%) were females. A total of 144 subjects participated in the first-dose group, nine $(6.3 \%)$ of whom developed protective antibody titre (8.3\% of males and $4.2 \%$ of females). Of the 121 participants in the seconddose group, $108(89.3 \%)$ developed protective antibody titre (98.3\% of males and $80.3 \%$ of females), while of the 111 participants in the third-dose group, $100 \%$ protectively sero-converted. Males were more likely to develop protective antibody titre than females after the second dose $(\mathrm{P}<0.05)$.

Conclusion: This data provides additional evidence for the efficacy of Shanvac-B rDNA hepatitis B vaccine and the need to adhere to the recommended three-dose schedule to achieve full and lasting sero-protection among Nigerians.
\end{abstract}

Key words: Shanvac-B; hepatitis B vaccine; HBV; sero-conversion; antibody titre

J Infect Dev Ctries 2010; 4(11):740-744.

(Received 09 January 2010 - Accepted 04 July 2010)

Copyright () 2010 Adoga et al. This is an open-access article distributed under the Creative Commons Attribution License, which permits unrestricted use, distribution, and reproduction in any medium, provided the original work is properly cited.

\section{Introduction}

Since the launch of the first universal hepatitis B vaccination programme for infants in July 1984 in Taiwan, infant immunisation programmes in many countries have led to marked decreases in the incidence and prevalence of heptatitis B among younger, vaccinated members of these populations [1-3]. However, a number of countries, most of which are in the developing world, such as Nigeria, are still grappling with a high incidence and prevalence of this silent-killer virus [4-6].

Recombinant hepatitis B vaccine was introduced in 1986 and has gradually replaced the plasmaderived hepatitis $\mathrm{B}$ vaccine. The active substance in recombinant hepatitis $\mathrm{B}$ vaccine is $\mathrm{HBsAg}$ that has been produced in yeast or mammalian cells into which the HBsAg gene (or HBsAg/pre-HBsAg genes) has been inserted using plasmids [7].

The endemicity of hepatitis B is described by the prevalence of $\mathrm{HBsAg}$ in the general population of a defined geographical area, and it varies considerably globally: HBsAg prevalences of $>8 \%$ are typical of highly endemic areas and prevalences of $2-7 \%$ are found in areas of intermediate endemicity, whereas in areas with low endemicity, $<2 \%$ of the population is HBsAg-positive [7].

More than two billion people alive today have had an HBV infection at some time in their lives. About 360 million have chronic infections and become carriers; these people are at a high risk of liver cirrhosis and primary liver cancer $[4,5,7]$. 
Due to the availability of effective vaccine and post-exposure prophylaxis, the Centre for Disease Control, USA recommends HBsAg testing for the following people: pregnant women; infants born to HBsAg-positive mothers; household contacts and sex partners of $\mathrm{HBV}$-infected persons; persons who are the source of blood or body fluid exposures that might warrant post-exposure prophylaxis (e.g. needle-stick injury to a health-care worker or sexual assault); persons born in geographical regions in which HBsAg prevalence is $\geq 2 \%$; men who have sex with men; injection-drug users; and HIV-infected individuals [2].

Globally, as of 2008, the hepatitis B vaccine has been incorporated as an integral part of the national infant immunization programmes in 177 countries , and an estimated $69 \%$ of the 2008 birth cohort received three doses of the hepatitis $B$ vaccine. Moreover, the protective efficacy of hepatitis B vaccination is related to the induction of anti-HBs antibodies, and also involves the induction of memory T-cells. An anti-HBs concentration of 10 $\mathrm{mIU} / \mathrm{mL}$ measured between one and three months after the administration of the last dose of the primary vaccination series is considered a reliable marker of protection against infection. The primary hepatitis B immunization series conventionally consists of three doses of vaccine. However, four doses may be given for programmatic reasons. Adverse events following immunization against hepatitis B are infrequent and generally mild [7].

Nigeria is a country highly endemic with hepatitis B in spite of a government HBV infant immunisation programme [5,6,8]. However, successful hepatitis B vaccination strategies have resulted in a dramatic reduction of HBV transmission in many countries with historically high endemicity [7]. Hence, the questions that develop are those that seek to examine the effectiveness of the Nigerian government's HBV vaccination programme and the efficacy of the vaccines used in the country.

The immunogenicity and safety of Shanvac-B hepatitis B vaccine has been demonstrated in comparison with some other commercially available hepatitis B vaccines (Genvac-B and Engerix-B) in infants born to $\mathrm{HBsAg}$-positive mothers and healthy adults in India (where Shanvac-B was developed) with good results [9-12]. Some other studies in India have also evaluated the immunogenicity of this vaccine with impressive outcomes [13-15].

However, though this vaccine is commonly used in Nigeria, there are no data on Nigerian subjects regarding its efficacy or lack thereof in terms of its immunogenicity. This study aimed to evaluate the rate of protective antibody titre induction in Nigerian subjects after vaccination with the Shanvac-B hepatitis B vaccine in a zero-, one- and six-month schedule.

\section{Methodology}

Study area and population

The study area comprised the Nasarawa State of Nigeria. Nasarawa State has an area of $27,117 \mathrm{Km}^{2}$ and a population of $1,863,275$. It is bounded in the north by Kaduna State, the west by Abuja (the capital of Nigeria), the south by the Kogi and Benue States, and the east by the Taraba and Plateau States [5]. A total of 376 subjects participated, from whom 144, 121 and 111 specimens were collected by venepuncture for the first, second and third dose analysis, respectively.

\section{Ethical considerations}

Signed informed consent was obtained from participants, parents, or legal representatives. Clearance was given by the Research Ethics Institutional Review Board of Innovative Biotech Ltd. in line with the code of ethics for biomedical research.

\section{Exclusion and inclusion criteria}

The study excluded persons who had an HBsAgpositive test, were anti-HBs positive, were over 50 years old, had an active illness or known immunodeficiency including TB or HIV/AIDS, had been administered immunoglobulins and bloodderived products in the last six months, had been treated with immunosuppressing agents including corticosteroids, or had evidence of skin infection or disease. Pregnant or lactating women were also excluded. Individuals who had no history of previous hepatitis $\mathrm{B}$ vaccination, who were $\mathrm{HBsAg}$-negative and anti-HBs negative, who showed no evidence of skin infection at any site, and who signed informed consent were included in the study.

\section{Shanvac-B hepatitis $B$ vaccine}

Shanvac B (Shantha Biotechnics Limited, Hyderabad, India), a recombinant vaccine containing a purified major surface antigen of the hepatitis $B$ virus was administered to selected subjects.

Adults were administered $1 \mathrm{ml}$ of the vaccine as the dose while children were given $0.5 \mathrm{ml}$ of the vaccine. The subjects recruited for this study were 
administered the vaccine on a zero-, one- and sixmonth schedule (schedule B), the schedule recommended for routine immunisation. Previous studies have shown the safety and efficacy of this vaccine $[9,10,12]$.

\section{Specimen collection and analysis}

Specimens were collected between January and September 2009. $2 \mathrm{~mL}$ blood samples were aseptically collected from each participant aged $\leq 50$ years. prior to the administration of the second dose of the vaccine (for the first-dose subject group), one month after the second dose (for the second-dose subject group), and one month after the third dose (for the last group). Sera were separated into cryovials at Innovative Biotech Ltd, and frozen at $21^{\circ} \mathrm{c}$ until analysed. Smart Check HBsAb (Globalemed, Alexandria VA, USA) was used for the detection of the protective antibody titre induction according to the manufacturer's instructions. Protective antibody titre was defined as a titre of $\geq$ $10 \mathrm{mIU} / \mathrm{mL}$.

\section{Statistical analysis}

Data were subjected to statistical analysis using the statistical software SPSS version 15.0 (SPSS Inc, Chicago, USA). Pearson's chi-squares and $P$ values were calculated at a $95 \%$ confidence limit.

\section{Results}

A total of 376 subjects participated. Of these, 192 $(51.1 \%)$ were males and $184(48.9 \%)$ were females. Out of 144 subjects (72 males and 72 females) who participated in the first-dose group of vaccines, nine (6.3\%) developed protective antibody titre $(8.3 \%$ of males and $4.2 \%$ of females). Of the 121 (60 males and 61 females) participants in the second-dose group, $108(89.3 \%)$ developed protective antibody titre $(98.3 \%$ of males and $80.3 \%$ of females). There was $100 \%$ protective antibody titre induction in all age groups among the 111 participants (60 males and 51 females) in the third-dose group. Those aged $\leq 10$ years were more likely to develop protective antibody titre than those in the older age groups (Table 2). Gender finding shows that males were significantly more likely to develop protective antibody titre than females after the second dose $(P=0.004$, Table 2$)$.

Table 1: Protective sero-conversion rate after first dose with Shanvac-B hepatitis B vaccine in Nigerians

\begin{tabular}{|l|l|l|l|l|l|l|}
\hline \multirow{2}{*}{$\begin{array}{l}\text { Age } \\
\text { years }\end{array}$} & \multirow{2}{*}{$\begin{array}{l}\text { № } \\
\text { Tested }\end{array}$} & \multicolumn{2}{|c|}{ Sex } & \multicolumn{2}{|c|}{ № positive } & $\begin{array}{l}\text { № / \% } \\
\text { Positive }\end{array}$ \\
\cline { 3 - 7 } & Male & Female & Male (\%) & Female (\%) & $1(8.3)$ & $3(13.6)$ \\
\hline$\leq 10$ & 22 & 10 & 12 & $2(20.0)$ & $2(11.1)$ & $6(16.7)$ \\
\hline $11-20$ & 36 & 18 & 18 & $4(22.2)$ & $0(0.0)$ & $0(0.0)$ \\
\hline $21-30$ & 24 & 13 & 11 & $0(0.0)$ & $0(0.0)$ & $0(0.0)$ \\
\hline $31-40$ & 29 & 16 & 13 & $0(0.0)$ & $0(0.0)$ & $0(0.0)$ \\
\hline $41-50$ & 33 & 15 & 18 & $0(0.0)$ & $3(4.2)$ & $9(6.3)$ \\
\hline Total & 144 & 72 & 72 & $6(8.3)$ & & \\
\hline
\end{tabular}

$P$ value $=1.000$

Table 2: Protective sero-conversion rate after second dose with Shanvac-B hepatitis B vaccine in Nigerians

\begin{tabular}{|l|l|l|l|l|l|l|}
\hline \multirow{2}{*}{$\begin{array}{l}\text { Age } \\
\text { (years) }\end{array}$} & \multirow{2}{*}{ № tested } & \multicolumn{4}{|l|}{ Sex } & \multicolumn{2}{|c|}{ № Positive } & $\begin{array}{l}\text { № / \% } \\
\text { Positive }\end{array}$ \\
\cline { 3 - 7 } & & Male & Female & Male (\%) & Female (\%) & $16(100)$ \\
\hline$\leq 10$ & 16 & 7 & 9 & $7(100)$ & $9(100)$ & $24(96.0)$ \\
\hline $11-20$ & 25 & 13 & 12 & $13(100)$ & $11(91.7)$ & $30(96.8)$ \\
\hline $21-30$ & 31 & 14 & 17 & $14(100)$ & $16(94.1)$ & $23(92.0)$ \\
\hline $31-40$ & 25 & 11 & 14 & $10(90.9)$ & $13(92.9)$ & $15(62.5)$ \\
\hline $41-50$ & 24 & 15 & 9 & $15(100)$ & $0(0.00)$ & $108(89.3)$ \\
\hline Total & 121 & 60 & 61 & $59(98.3)$ & $49(80.3)$ & \\
\hline
\end{tabular}

$P$ value $=0.004$ 


\section{Discussion}

An effective vaccine against hepatitis B has been available since 1982 [16] and in 1993, the World Health Organization made a recommendation for the inclusion of hepatitis B vaccination in the Expanded Programme of Immunisation (EPI) of all countries by the year 1997 [12]. This initiative has led to a significant reduction in the incidence of hepatitis B virus infections in most countries that complied.

In this study, the rate of protective sero-conversion against hepatitis B virus infections among Nigerian subjects following a zero-, one- and six-month schedule of vaccination with Shanvac-B hepatitis B vaccine was investigated. After the first, second and third doses of vaccinations, protective seroconversion rates were $6.3 \%, 89.3 \%$ and $100 \%$ respectively.

Primary immunisation with hepatitis B vaccine can take either schedule A or schedule B. While schedule $\mathrm{A}$ is recommended for high-risk people, schedule $B$ is recommended for routine immunisation. In schedule $\mathrm{A}$, the vaccine is given at zero, one and two months, while in schedule B it is given at zero, one and six months [10]. In schedule A, a booster dose is recommended 12 months after the first dose, and a second booster may be required after eight years if the titre falls below $10 \mathrm{mIU} / \mathrm{mL}$. In schedule $\mathrm{B}$, a booster may be required after eight to ten years if the titre falls below $10 \mathrm{mIU} / \mathrm{mL}$ [7].

The $100 \%$ sero-protectivity achieved one month after the third dose in this study is consistent with previous findings. For instance, protective seroconversion rates that range from $96.4 \%$ to $100 \%$ have been reported [9-15]. However, the $6.3 \%$ protective sero-conversion rate one month after the first dose was lower than previous reports of $22 \%, 22.4 \%$ and $49.1 \%$ in India $[11,13,14]$. The reason for this difference is unclear.

However, a further evaluation of this observation with a larger sample size among Nigerians is recommended as it will offer a clearer understanding of whether or not sero-protectivity after a first dose of vaccination with Shanvac-B varies with race or region. The $89.3 \%$ sero-protectivity rate achieved one month after the second dose of the vaccine is in accord with previous findings of $68.9 \%, 77 \%, 86.9 \%$ and $99 \%$ in Indian subjects [11, 13-15].

The results of the current study showed that those aged $\leq 10$ years were more likely to develop protective antibody titre $(\geq 10 \mathrm{mIU} / \mathrm{mL})$ one month after second dose of vaccination than those in the older age groups (Table 2). Since infants fall within this age group, this finding supports previous reports that found a $100 \%$ sero-protectivity rate among infants who completed the three-dose regimen, with one of the studies reporting a comparative $96.4 \%$ rate in adults $[9,10]$.

Males were significantly more likely to be seroprotected one month after the second dose of vaccination than females (Table $2, P=0.004$ ). A previous investigation reported a higher geometric mean titre (GMT) in females than males after a threedose regimen [11]. However, the present finding resulted after a second dose while the other was GMT after the third dose. While there is no clear explanation for this discrepancy, future studies could further examine this question.. The present study was qualitative, not quantitative, hence making the evaluation of GMT for both genders impossible. Usually, the arithmetic values of anti-HBs are converted as GMT of anti-HBs [12].

Since the evaluation of GMT will not be possible in any qualitative investigation, we recommend that future similar studies should be quantitative in approach to achieve a better understanding of the anti-HBs induced and to provide a better basis for gender comparison. Future similar investigations should also consider a follow-up study with a larger sample size involving the same subjects, since this factor was not part of our study design.

Since the cost of Shanvac-B is almost $50 \%$ lower than that of other brands of hepatitis B vaccine [10], and its efficacy has been confirmed by some studies without a significant difference in its immunogenicity in comparison with the other types [9, 11, 12], this vaccine might be considered as the one of choice in all countries where resources are limited, including Nigeria.

A cost effectiveness study on HBV vaccination in Nigeria is therefore recommended. However, for many developing countries, the implementation of a universal immunisation programme against hepatitis B is probably cost effective in many settings [17]. Studies indicate that vaccinating newborn infants against hepatitis B is cost effective in countries with a low prevalence, intermediate prevalence and high prevalence of hepatitis B [7]. Such study in the Gambia showed that when compared with no intervention, a hepatitis B vaccination programme would cost US \$28 per disability-adjusted life year (DALY) averted from the societal perspective, or US $\$ 47$ per DALY averted from the payer's perspective. Comparable results have been found in Mozambique [7]. 
This study provides important data about the efficacy of Shanvac-B recombinant DNA hepatitis B vaccine among Nigerians and the need to adhere to the recommended three-dose schedule to achieve full and lasting sero-protection.

\section{Acknowledgements}

We are indebted to Innovative Biotech Ltd for sponsoring this work.

\section{References}

1. Liang X, Bi S, Yang W, Wang L, Cui G, Cui F, Zhang Y, Liu J, Gong X, Chen Y, Wang F, Zheng H, Wang F, Guo J, Jia Z, Ma J, Wang H, Luo H, Li L, Jin S, Hadler SC, Wang Y (2009) Epidemiological serosurvey of hepatitis B in China - Declining HBV prevalence due to hepatitis B vaccination. Vaccine 27: 6550-6557.

2. Weinbaum CM, Williams I, Mast EE, Wang SA, Finelli L, Wasley A, Neitzel SM, Ward JW (2008) Recommendations for identification and public health management of persons with chronic hepatitis B virus infection. MMWR 57: 1-20.

3. Shuler CM, Fiore AE, Neeman R, Bell BP, Kuhnert W, Watkins S, Kilgour K, Arnold KE (2009) Reduction in hepatitis B virus Seroprevalence among U.S.-born children of foreign-born Asian parents - benefit of universal infant hepatitis B vaccination. Vaccine 27: 5942-5947.

4. Bwogi J, Braka F, Makumbi I, Mishra V, Bakamutumaho B, Nanyunja M, Opio A, Downing R, Biryahwaho B, Lewis RF (2009) Hepatitis B infection is highly endemic in Uganda: findings from a national serosurvey. Afr Health Sci 9: 98108.

5. Adoga MP, Banwat EB, Forbi JC, Nimzing L, Pam CR, Gyar SD, Agabi YA, Agwale SM (2009) Human immunodeficiency virus, hepatitis B virus and hepatitis C virus: sero-prevalence, co-infection and risk factors among prison inmates in Nasarawa State, Nigeria. J Infect Dev Ctries 3: 539-547.

6. Forbi JC, Onyemauwa N, Gyar SD, Oyeleye AO, Entonu P, Agwale SM (2008) High prevalence of hepatitis B virus among female sex workers in Nigeria. Rev Inst Med trop S Paulo 50: 219-221.

7. World Health Organisation (2009). Position Paper on Hepatitis B Vaccines. Weekly Epidemiological Record; 40: 405-420. Available at: http://www.who.int/wer. Accessed 7 March 2010.

8. Egah DZ, Banwat EB, Audu ES, Iya D, Mandong BM, Anele AA, Gomwalk NE (2007) Hepatitis B surface antigen, hepatitis $\mathrm{C}$ and HIV antibodies in a low-risk blood donor group, Nigeria. East Mediterr Health J 13: 961-966.

9. Velu V, Nandakumar S, Shanmugam S, Jadhav SS, Kulkarni PS, Thyagarajan SP (2007) Comparison of three different recombinant hepatitis B vaccines: GeneVac-B, Engerix-B and Shanvac-B in high risk infants born to $\mathrm{HBsAg}$ positive mothers in India. World J Gastroenterol 13: 3084-3089.

10. Joshi N, Kumar A, Raghu MB, Bhave S, Arulprakash R, Bhusari P, Rao R (2004) Immunogenicity and safety of hepatitis B vaccine (Shanvac-B) using a novel pre -filled single use injection device Uniject in Indian subjects. Indian J Med Sci 58: 472-477.

11. Joshi N, Kumar A, Sreenivas DV, Palan S, Nagarjuna Kumar YR (2000) Safety and immunogenicity of indigenous recombinant hepatitis B vaccine (Shanvac-B ) in comparison with commercially available vaccine. Indian J Gastroenterol 19: 71-73.

12. Vijayakumar V, Hari R, Parthiban R, Mehta J, Thyagarajan SP (2004) Evaluation of immunogenicity and safety of Genevac-B: A new recombinant hepatitis B vaccine in comparison with Engerix-B and Shanvac-B in healthy adults. Indian J Med Microbiol 22: 34-38.

13. Murhekar MV, Murhekar KM, Arankalle VA, Sehgal SC (2002) Immune response to an indigenously developed hepatitis-B (Shanvac-B) vaccine in a tribal community of India. Vaccine 20: 3431-3435.

14. Kumar A, Joshi N, Sreenivas DV, Palan S, Nagarjuna Kumar YR (1998) Clinical safety and efficacy of indigenous recombinant hepatitis B vaccine. J Assoc Physicians India 46: 620-622.

15. Abraham P, Mistry FP, Bapat MR, Sharma G, Reddy GR, Prasad KS, Ramanna V (1999) Evaluation of a new recombinant DNA hepatitis B vaccine (Shanvac-B). Vaccine 17: $1125-1129$.

16. World Health Organisation. Hepatitis B. Fact Sheet $N^{0} 204$. Available: http://www.who.int/csr/disease/hepatitis. Accessed August 7th, 2009.

17. Tu HA, Woerdenbag HJ, Kane S, Riewpaiboon A, Van Hulst M, Postma MJ (2009) Economic evaluations of hepatitis B vaccination for developing countries. Expert Rev Vaccines 8: 907-920.

\section{Corresponding author}

Simon M. Agwale, PhD

Innovative Biotech USA Inc

Frederick Innovative Technology Center

4539 Metropolitan Ct, Frederick MD 21704, US

sagwale@innovativebiotechng.com, adogamoses@yahoo.com

Conflict of interests: No conflict of interests is declared. 